LE JOURNAL DE PHYSIQUE-LETTRES

Classification

Physics Abstracts

$02.30 \mathrm{H}$

\title{
Stable cycles with complicated structure
}

\author{
J. M. Gambaudo $\left({ }^{1}\right)$, P. A. Glendinning $\left({ }^{2}\right)$ and C. Tresser $\left({ }^{3}\right)$ \\ (1) L.A. 168, Parc Valrose, 06034 Nice Cedex, France \\ ( $\left.{ }^{2}\right)$ D.A.M.T.P., Silver Street, Cambridge CB3 9EW, U.K. \\ $\left({ }^{3}\right)$ L.A. 190, Parc Valrose, 06034 Nice Cedex, France
}

(Reçu le 3 mai 1985, accepté le 12 juin 1985)

\begin{abstract}
Résumé. - Dans des expériences où deux cycles stables se " collent " pour former un grand cycle stable (qui est essentiellement la combinaison des deux premiers) quand on varie un paramètre, de petites perturbations peuvent donner lieu à des cycles stables ayant une structure compliquée. Nous décrivons ce phénomène et indiquons quelle peut être la structure de ces orbites.

Abstract. - In experiments where two stable cycles come together to form one large stable cycle (which is essentially a combination of the first two) as a parameter is varied, small perturbations can lead to stable cycles with complicated structures. We describe this phenomenon and indicate the possible form of these complicated orbits.
\end{abstract}

Consider an experiment which produces, asymptotically, a periodic signal $X_{0}(t)$, and such that small perturbations give asymptotically the same signal, up to a translation in time. This suggests that the underlying dynamical system has a stable periodic orbit. Now suppose that for some sufficiently large perturbations of the initial conditions the experiment yields a periodic signal $X_{1}(t)$ which is not a time translation of $X_{0}(t)$ but which again corresponds to a stable periodic orbit. This situation can occur, for example, if the underlying dynamical system is invariant under some symmetry.

Although we intend to describe much more general cases, such symmetry conditions arise frequently in physical models and we shall start by describing a typical scenario which can be observed by varying a parameter in an experimental setting corresponding to a symmetric model.

Suppose :

(H1) The output can be chosen so that the signals $X_{0}(t)$ and $X_{1}(t)$ corresponding to symmetric initial conditions are not time translations of each other.

(H2) Consider an initial condition which gives asymptotically the signal $X_{i}(t), i=0,1$. If the parameter is varied slowly enough the new signal, $X_{i}^{\prime}(t)$, has essentially the same profile except that a longer time is spent in an almost stationary phase at the end of each period. 
(H3) As the parameter is varied further an abrupt change of the signal is observed, giving a new signal which is a regular alternation between the profiles of $X_{0}$ and $X_{1}$ (see Fig. 1).

For simplicity we shall say that the signals $X_{0}(t)=s_{0} s_{0} s_{0} \ldots$ and $X_{1}(t)=s_{1} s_{1} s_{1} \ldots$ have been " glued together " to give the new signal $s_{0} s_{1} s_{0} s_{1} \ldots$

Let us now return to the underlying dynamical system and denote by $\mathrm{C}_{0}$ and $\mathrm{C}_{1}$ the stable periodic orbits which correspond to the signals $X_{0}(t)$ and $X_{1}(t)$ respectively. The change of behaviour described above indicates that $C_{0}$ and $C_{1}$ come closer and closer to the same stationary point until they form a pair of homoclinic curves and are destroyed, giving rise to a new stable periodic orbit which essentially follows successively the loci in phase space of the cycles $C_{0}$ and $C_{1}$.

The aim of this paper is to describe the signals which can be generated when two stable cycles come close to the same stationary point in more general systems (i.e. the symmetry condition will be dropped).

Before proceeding we need to define sequences written-with two symbols which are rotation compatible. Let $\mathrm{S}$ stand for the set of all sequences written with the symbols $s_{0}$ and $s_{1}$.

$$
\mathrm{S}=\left\{\left(s_{0}^{m_{1}} s_{1}^{m_{2}} s_{0}^{m_{3}} s_{1}^{m_{4}} \ldots\right) ; m_{1} \geqslant 0, m_{2} \geqslant 0, m_{3}, \ldots,>0\right\} .
$$

We will say that an element of $S$ is rotation compatible with rotation number $\omega \in(0,1)$ if there is a series of numbers $n_{i}$ so that

$$
\begin{aligned}
& n_{0} \in[0,1] \\
& n_{i}=n_{i-1}+\omega(\bmod 1)
\end{aligned}
$$

and such that the $m_{1}$ first $n_{i}$ 's are greater than $1-\omega$, the $m_{2}$ following ones are smaller than $1-\omega$, the $m_{3}$ following these are greater than $1-\omega$, etc.

These sequences have many nice properties [5]; here we mention only the simplest of these : either

- the $m_{i}$ 's with $i$ odd are all of the form $N_{0}$ or $N_{0}+1$ for some $N_{0}>1$ (except perhaps for $m_{1}$ which can be smaller than $N_{0}$ ).

$-m_{i}=1$ for $i$ even

or of the same form, but with the roles of $s_{0}$ and $s_{1}$ exchanged.

This is a direct consequence of the form of the map $\theta^{\prime}=\theta+\omega(\bmod 1)$ when represented as a map of the unit interval. Note that $\omega$ gives the proportion of $s_{0}$ 's in the sequence and if $\omega$ is rational the sequence $s_{1}^{m_{2}} s_{0}^{m_{3}} \ldots$ is periodic.
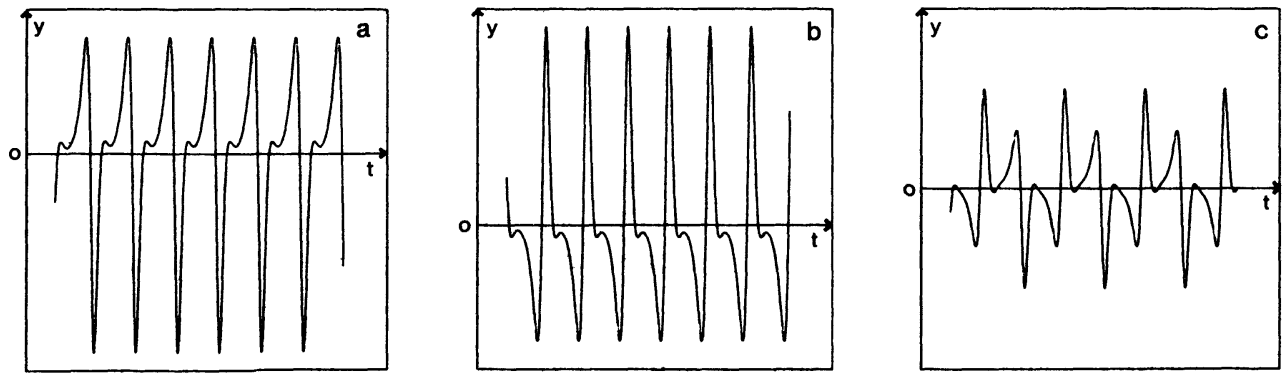

Fig. 1. - Signals obtained by numerical simulation of the example of figure 2 with $\varepsilon=0$ (which is invariant under a simple symmetry) illustrating a gluing bifurcation by plotting $y$ against time. (a) $\mu=0.53$ : a stable cycle; (b) $\mu=0.53$ : another stable cycle which is the image of (a) under the symmetry; (c) $\mu=0.534$ : a symmetric signal obtained by " gluing together " the basic signals of (a) and (b). 
If a physical system, controlled by two parameters $\mu_{0}$ and $\mu_{1}$ is such that $\left(^{1}\right)$

- for $\mu_{0}<0$ and $\mu_{1}<0$, there are two stable periodic cycles $\mathrm{C}_{0}\left(\mu_{0}, \mu_{1}\right)$ and $\mathrm{C}_{1}\left(\mu_{0}, \mu_{1}\right)$ giving, respectively, the signals $X_{0}\left(\mu_{0}, \mu_{1}, t\right)=s_{0} s_{0} s_{0} \ldots$ and $X_{1}\left(\mu_{0}, \mu_{1}, t\right)=s_{1} s_{1} s_{1} \ldots$ (depending on the initial conditions) for the measurement of the same quantity.

- for $i=0,1, \mu_{i} \rightarrow 0^{-}$, the signal $X_{i}\left(\mu_{1}, \mu_{2}, t\right)$ remains essentially unchanged except for longer and longer almost stationary phases between the pattern $s_{0}$ or $s_{1}$ which reappear periodically (i.e. the cycle $\mathrm{C}_{i}\left(\mu_{0}, \mu_{1}\right)$ approaches an equilibrium).

- in the region $\mu_{0}>0, \mu_{1}>0$, arbitrarily small $\mu_{0}$ and $\mu_{1}$ can be chosen so that there is a stable signal of the form $s_{0} s_{1} s_{0} s_{1} \ldots$

then it is likely that the theorems proved in [4] are applicable. This means that for parameter values near $\mu_{0}=\mu_{1}=0$ there are either 0,1 or 2 periodic signals which remain close to $C_{0} \cup C_{1}(0,0)$, and any periodic signal which exists is stable. Furthermore, all such periodic orbits can be described by rotation compatible sequences and if two stable signals $Y_{i}\left(\mu_{0}, \mu_{1}, t\right) i=0,1$ exist at the same parameter values then the rotation numbers, $p_{i} / q_{i}$, written in irreducible form associated with the rotation compatible sequences of $Y_{i}$ satisfy the condition $\left|p_{0} q_{1}-p_{1} q_{0}\right|=1$ (i.e. $p_{0} / q_{0}$ and $p_{1} / q_{1}$ are neighbours in some Farey series). Note also that in some cases there are parameter values for which there is a stable signal whose rotation compatible sequence has an irrational rotation number, so the signal is aperiodic, but not chaotic. In particular there exist volume-contracting flows in $\mathbb{R}^{3}$ with an attracting quasiperiodic orbit (technically this is the inverse limit of a Cherry flow on a torus with a hole). This is possible since quasiperiodic orbits which occur near the double homoclinic loop do not lie on an invariant torus.

As stated above, these results are not strictly valid. However, they can be proved rigorously for sufficiently smooth perturbations of ordinary differential equations with a pair of trajectories which are biasymptotic to the same stationary point, 0 , providing that the characteristic equation of the linearized flow near 0 has a simple real positive eigenvalue, $\lambda$, and all the other eigenvalues are to the left of $-\lambda$, in the complex plane [4]. The conditions given here provide strong evidence that the underlying dynamical system which gives rise to the observed signal is indeed of this form. In particular, the condition that the signal remains stable even when it has long almost stationary phases suggests strongly that the characteristic equation at the stationary point (for the underlying dynamical system) is of the form given above [6].

These very general results can be made more explicit given more information about the underlying dynamical system : the linearized flow near $0 \mathrm{can}$, in general, be written in one of two forms (after a near-identity change of coordinates)

$$
\begin{aligned}
\mathrm{L}_{1}: \dot{x}_{1} & =\lambda_{1} x_{1} \\
\dot{x}_{2} & =-\lambda_{2} x_{2}
\end{aligned}
$$

$$
\begin{aligned}
\mathrm{L}_{2}: \dot{x}_{1} & =\lambda_{1} x_{1} \\
\dot{x}_{2} & =-\lambda_{2} x_{2}+\omega x_{3} \\
\dot{x}_{3} & =-\omega x_{2}-\lambda_{2} x_{3}
\end{aligned}
$$

with $0<\lambda_{1}<\lambda_{2}$. The dots indicate variables with eigenvalues of the linearized flow to the left of $-\lambda_{2}$. These two cases can usually be distinguished by a close inspection of the almost stationary phase of the signal (whose length, in both cases, diverges as the log of the distance of the parameter from the bifurcation point). This stationary phase will be flat if the underlying dynamical system is

( $\left.{ }^{1}\right)$ Although this is not the general case, the reader can consider that the parameters $\mu_{0}$ and $\mu_{1}$ are such that the system is invariant under some symmetry when $\mu_{0}=\mu_{1}$. 

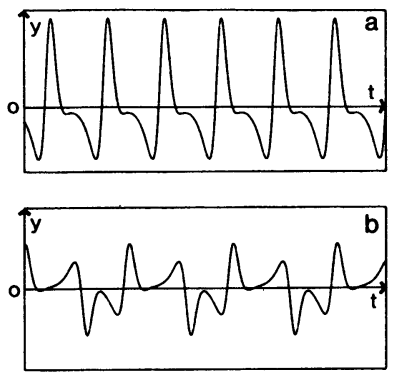
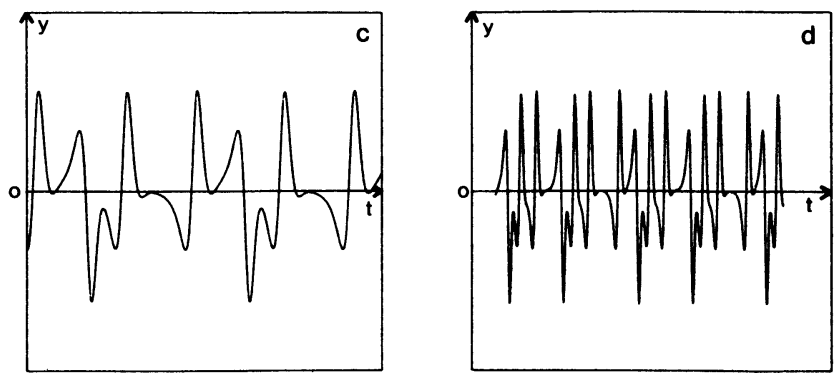

Fig. 2. - Plots of $y$ against time for the system $\dot{x}=y, y=z+\varepsilon x^{2}, \dot{z}=-z-0.5 y+\mu x\left(1-x^{2}\right)$ showing some stable cycles. The parameter values of (c) and (d) may be outside the rigorous domain of validity of the analysis. (a) $\mu=0.54, \varepsilon=0.005:$ a stable cycle with rotation number 1 , and symbol $s_{1} s_{1} s_{1}$ $s_{1} s_{1} s_{1} \ldots$ (b) $\mu=0.54, \varepsilon=0.005$ : another stable cycle with rotation number $1 / 2$ which coexists with the cycle shown in (a). The symbol is $s_{1} s_{0} s_{1} s_{0} s_{1} s_{0} s_{1} \ldots$ (c) $\mu=0.556, \varepsilon=0.008:$ a stable cycle with rotation number 2/3. The symbol is $s_{1} s_{0} s_{1} s_{1} s_{0} s_{1} \ldots$ (d) $\mu=0.556, \varepsilon=0.01:$ a stable cycle with rotation number 2/7. The symbol is $s_{0} s_{1} s_{1} s_{0} s_{1} s_{1} s_{1} s_{0} s_{1} s_{1} s_{0} s_{1} s_{1} s_{1} s_{0} s_{1} \ldots$

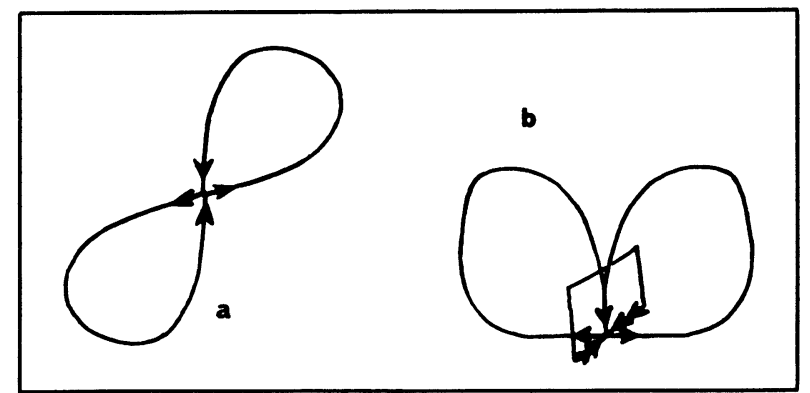

Fig. 3. - The figure eight (a) and the butterfly (b).
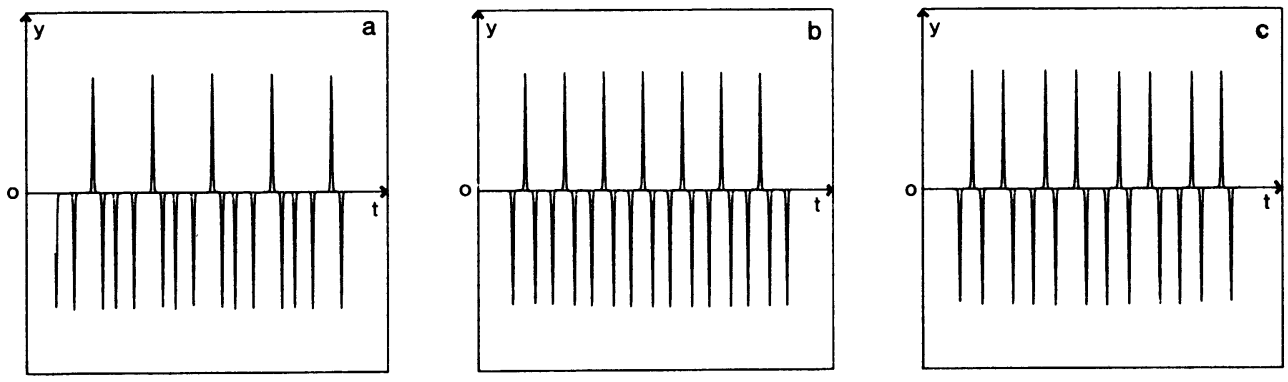

Fig. 4. - Plots of $y$ against time for a perturbation of the system described in [1] : $\dot{x}=1.8(x-y)+\varepsilon x^{2}$, $\dot{y}=-7.2 y+x z+\mu x^{3}, \dot{z}=-2.7 z+x y-0.07 z^{2}$ showing some stable cycles with $\varepsilon=0.001$. Note that the basic signal is a little different from that of the previous figures and that the associated rotation numbers vary monotonically with $\mu$. (a) $\mu=0.07521$ : rotation number $1 / 4$. The symbol is $s_{0} s_{0} s_{1} s_{0} s_{0} s_{0}$ $s_{1} s_{0} s_{0} s_{0} s_{1} s_{0} s_{0} s_{0} s_{1} s_{0} s_{0} s_{0} s_{1} s_{0} \ldots$ (b) $\mu=0.07502$ : rotation number $1 / 3$. The symbol is $s_{0} s_{1} s_{0} s_{0} s_{1}$ $s_{0} s_{0} s_{1} s_{0} s_{0} s_{1} s_{0} s_{0} s_{1} s_{0} s_{0} s_{1} s_{0} s_{0} s_{1} s_{0} s_{0} \ldots$ (c) $\mu=0.075014$ : rotation number $2 / 5$. The symbol is $s_{0} s_{1} s_{0} s_{1} s_{0} s_{0} s_{1} s_{0} s_{1} s_{0} s_{0} s_{1} s_{0} s_{1} s_{0} s_{0} s_{1} s_{0} s_{1} s_{0} \ldots$ 
of $\mathrm{L}_{1}$-type and will have small oscillations if of $\mathrm{L}_{2}$-type (corresponding to spiralling in the eigenplane of the complex pair of eigenvalues).

In the complex case $\left(\mathrm{L}_{2}\right)$ signals with any rotation number $\omega \in[0,1]$ can be found in a neighbourhood of $\mu_{0}=\mu_{1}=0$, and periodic signals coexist at some parameter value if and only if their irreducible rotation numbers $p_{0} / q_{0}$ and $p_{1} / q_{1}$ satisfy $\left|p_{0} q_{1}-p_{1} q_{0}\right|=1$ [3] (see Fig. 2).

In the real case $\left(\mathrm{L}_{1}\right)$ there are two different configurations of the homoclinic curves. Both homoclinic loops may return on the same branch of $x_{2}$ (although this is clearly impossible in $\mathbb{R}^{2}$ ) or they may return on opposite branches. These configurations are referred to as the butterfly and figure eight respectively (see Fig. 3). More precise results can be obtained by specifying which of these two configurations is observed. These will be reported elsewhere. We just mention here that in general, in the real cases, one has super selection rules, either on the possible rotation numbers (think of the two dimensional case !) or on the possible pairs.

Figure 4 shows some stable orbits observed in a system with a butterfly configuration. (This system is a non-symmetric perturbation of the example in [1]).

\section{Acknowledgements.}

We would like to thank the King's College Research Center for its hospitality. P.A.G. was supported by an S.E.R.C. studentship.

\section{References}

[1] Arnéodo, A., Coullet, P. and Tresser, C., Phys. Lett. A 81 (1981) 197.

[2] Coullet, P., Gambaudo, J. M. and Tresser, C., C.R. Acad. Sci. Paris, Sér. I 299 (1984) 253.

[3] Gambaudo, J. M., Glendinning, P. and Tresser, C., C.R. Acad. Sci. Paris, Sér. I 299 (1984) 711.

[4] Ġambaudo, J. M., Glendinning, P.and Tresser, C., The gluing bifurcation: I. Symbolic dynamics of the closed curves preprint, Université de Nice (1985).

[5] Gambaudo, J. M., Lanford III, O. and Tresser, C., C.R. Acad. Sci. Paris, Sér. I 299 (1984) 823.

[6] ŜI'NIKov, L. P., Math. USSR Sbornik 6 (1968) 427. 